\title{
On the use of pseudocontact shifts in the structure determination of metalloproteins ${ }^{\dagger}$
}

\author{
Malene Ringkjøbing Jensen, ${ }^{1}$ D. Flemming Hansen, ${ }^{1}$ Umit Ayna, ${ }^{2}$ Robert Dagil, ${ }^{1}$ Mathias A. \\ S. Hass, ${ }^{1}$ Hans E. M. Christensen ${ }^{3}$ and Jens J. Led ${ }^{1 *}$
}

${ }^{1}$ Department of Chemistry, University of Copenhagen, Universitetsparken 5, DK-2100 Copenhagen $\varnothing$, Denmark

2 Department of Chemistry, Bilkent University, 06800 Ankara, Turkey

${ }^{3}$ Department of Chemistry, The Technical University of Denmark, Building 207, DK-2800 Kgs. Lyngby, Denmark

Received 3 October 2005; Revised 13 November 2005; Accepted 15 November 2005

\begin{abstract}
The utility of pseudocontact shifts in the structure refinement of metalloproteins has been evaluated using a native, paramagnetic $\mathrm{Cu}^{2+}$ metalloprotein, plastocyanin from Anabaena variabilis (A.v.), as a model protein. First, the possibility of detecting signals of nuclei spatially close to the paramagnetic metal ion is investigated using the WEFT pulse sequence in combination with the conventional TOCSY and ${ }^{1} \mathrm{H}-{ }^{15} \mathrm{~N}$ HSQC sequences. Second, the importance of the electrical charge of the metal ion for the determination of correct pseudocontact shifts from the obtained chemical shifts is evaluated. Thus, using both the $\mathrm{Cu}^{+}$plastocyanin and $\mathrm{Cd}^{2+}$-substituted plastocyanin as the diamagnetic references, it is found that the $\mathrm{Cd}^{2+}$-substituted protein with the same electrical charge of the metal ion as the paramagnetic $\mathrm{Cu}^{2+}$ plastocyanin provides the most appropriate diamagnetic reference signals. Third, it is found that reliable pseudocontact shifts cannot be obtained from the chemical shifts of the ${ }^{15} \mathrm{~N}$ nuclei in plastocyanin, most likely because these shifts are highly dependent on even minor differences in the structure of the paramagnetic and diamagnetic proteins. Finally, the quality of the obtained ${ }^{1} \mathrm{H}$ pseudocontact shifts, as well as the possibility of improving the accuracy of the obtained structure, is demonstrated by incorporating the shifts as restraints in a refinement of the solution structure of A.v. plastocyanin. It is found that incorporation of the pseudocontact shifts enhances the precision of the structure in regions with only few NOE restraints and improves the accuracy of the overall structure. Copyright $\subset 2006$ John Wiley \& Sons, Ltd.
\end{abstract}

KEYWORDS: NMR; ${ }^{1} \mathrm{H} ;{ }^{13} \mathrm{C} ;{ }^{15} \mathrm{~N}$; paramagnetic metalloprotein; blue copper protein; plastocyanin; pseudocontact shift; WEFT; SERF

\section{INTRODUCTION}

Paramagnetic metal ions are potential tools in the structure determination of proteins. In paramagnetic metalloproteins, the dipolar interactions between the unpaired electrons of the metal ion and the nuclei of the protein contain

${ }^{\dagger}$ Dedicated to Professor David M. Grant of the University of Utah on the occasion of his 75th birthday, in recognition of the outstanding contributions he has made to the methodology of nuclear magnetic resonance and its application to a wide range of chemical topics over a long period of time.

${ }^{*}$ Correspondence to: Jens J. Led, Department of Chemistry, University of Copenhagen, Universitetsparken 5, DK-2100 Copenhagen Ø, Denmark. E-mail: led@kiku.dk

Contract/grant sponsor: Danish Natural Science Research Council Contract/grant numbers: 9400351, 9801801, 51-00211, 21-01-0545, and 21-04-0519.

Contract/grant sponsor: Carlsbergfondet; Contract/grant number: $1624 / 40$.

Contract/grant sponsor: Novo Nordisk Fonden; Contract/grant number: 2003-11-28

Contract/grant sponsor: Villum Kann Rasmussen Fonden;

Contract/grant number: 8.12.2003. valuable long-range structure information. In principle, these interactions can be detected by NMR spectroscopy through enhanced nuclear relaxation rates and changes in chemical shifts, i.e. the pseudocontact shifts.

However, spin diffusion may complicate the use of paramagnetic relaxation enhancements in structure refinements of proteins (Hansen DF, Led JJ, unpublished results). Furthermore, fast exchange with solvent water may obscure the paramagnetic relaxation enhancement of amide protons. ${ }^{1-3}$ In contrast, the pseudocontact shifts remain unaffected by these phenomena. Also, the pseudocontact shifts are experimentally more accessible, and can be determined accurately if an appropriate diamagnetic reference can be obtained. Recently, long-range pseudocontact shift restraints have been used as a supplement to the conventional nuclear Overhauser enhancements (NOEs) and dihedral angle restraints ${ }^{4}$ in structure refinements of metalloproteins, and proteins and nucleic acids with artificially incorporated paramagnetic metal ions. ${ }^{5-11}$ Thus, the pseudocontact shifts have proved valuable in the refinement of structures where a sufficient number of NOEs are difficult to obtain. Also, pseudocontact shifts have been used to determine the relative orientation 
of protein molecules in intermolecular complexes such as plastocyanin and cytochrome $f .{ }^{12}$

The pseudocontact shifts of nuclei close to the metal ion in paramagnetic proteins are particularly useful since they carry information about the protein structure close to the metal site. This information is difficult to obtain by the normal NMR approach for structure determination of proteins, where the strong electron-nuclear interaction often prevents the observation of the NOEs that are essential for this approach. Yet, spectral overlap and broadening of the signals from nuclei spatially close to the paramagnetic metal ion may also prevent observation of the latter signals by standard NMR techniques. However, as demonstrated here, a combination of the water eliminated Fourier transform $(\text { WEFT })^{13}$ pulse sequence both with the ${ }^{1} \mathrm{H}-{ }^{15} \mathrm{~N}$ HSQC and the TOCSY sequences can recover some of these signals by suppressing the strong signals arising from nuclei more distant from the paramagnetic metal ion.

Here, we investigate the applicability of pseudocontact shifts in the determination of the solution structure of paramagnetic metalloproteins. The blue copper protein, Anabaena variabilis (A.v.) plastocyanin, which functions as an electron carrier in the electron transfer process of the photosynthesis, was used as a model protein. In particular, we explore the possibility of measuring the pseudocontact shifts of paramagnetically broadened signals from nuclei relatively close to the paramagnetic metal ion. This possibility is important especially in the case of paramagnetic copper proteins, in which the signals are broad because of a relatively long electron relaxation time, and the pseudocontact shifts are small because of a relatively small anisotropy of the g-tensor. ${ }^{14}$ Furthermore, we examine the importance of choosing an appropriate diamagnetic reference, and the effect of minor structural changes on the measured ${ }^{15} \mathrm{~N}$ pseudocontact shifts. Finally, we investigate the impact of the ${ }^{1} \mathrm{H}$ pseudocontact shifts on the obtained solution structure, when the shifts are included as restraints in the structure determination of A.v. plastocyanin.

\section{THEORY}

\section{Pseudocontact shifts}

In paramagnetic proteins, the observed chemical shifts, $\delta$, of the protein nuclei are given by

$$
\delta=\delta_{\text {dia }}+\delta_{\text {con }}+\delta_{\text {pcs }}
$$

Here, $\delta_{\text {dia }}$ is the diamagnetic chemical shift and $\delta_{\text {con }}$ is the Fermi contact shift originating from the scalar coupling with the unpaired electrons of the paramagnetic metal ion. Normally, the contact shift is observed only for nuclei within a few bonds from the paramagnetic center. Furthermore, $\delta_{\text {pcs }}$ is the pseudocontact shift caused by the dipolar interaction with the unpaired electrons of the paramagnetic metal ion. The pseudocontact shift is given by ${ }^{15}$

$$
\delta_{\mathrm{pcs}}=\frac{\mu_{0} \mu_{\mathrm{B}}^{2} S(S+1)}{36 \pi k T r^{3}}\left[g_{\mathrm{ax}}\left(3 \cos ^{2} \theta-1\right)+\frac{3}{2} g_{\mathrm{eq}} \sin ^{2} \theta \cos (2 \phi)\right]
$$

Here, $\mu_{0}$ is the permeability of vacuum, $\mu_{\mathrm{B}}$ is the Bohr magneton, $S$ is the electron spin number $\left(1 / 2\right.$ for $\left.\mathrm{Cu}^{2+}\right), k$ is the Boltzmann constant, and $T$ is the absolute temperature. Furthermore, $(r, \theta, \phi)$ are the spherical coordinates of the nucleus in the principal coordinate system of the $g$-tensor. Finally, the anisotropic $g$-tensor components, $g_{\text {ax }}$ and $g_{\text {eq }}$, are given by

$$
\begin{aligned}
& g_{\mathrm{ax}}=g_{z z}^{2}-0.5\left(g_{x x}^{2}+g_{y y}^{2}\right) \\
& g_{\mathrm{eq}}=g_{x x}^{2}-g_{y y}^{2}
\end{aligned}
$$

where $g_{x x}, g_{y y}$, and $g_{z z}$ are the principal $g$-values. To take into account the experimentally measured pseudocontact shifts in a structure refinement, the size and orientation of the $g$-tensor must be known. The $g$-tensor parameters can be evaluated in the structure refinement, as described below, or independently by single-crystal EPR experiments.

\section{EXPERIMENTAL}

\section{Overexpression and purification of ${ }^{15} \mathrm{~N}$-labeled A.v. plastocyanin}

Uniformly ${ }^{15} \mathrm{~N}$-labeled plastocyanin from Anabaena variabilis (A.v.), Nostoc PCC7937, was expressed and purified as described previously. ${ }^{16}$ The protein was dissolved in $10 \%$ $\mathrm{D}_{2} \mathrm{O} / 90 \% \mathrm{H}_{2} \mathrm{O}$ or $99.99 \% \mathrm{D}_{2} \mathrm{O}$ with $100 \mathrm{~mm} \mathrm{NaCl}$.

\section{Overexpression and purification of}

\section{$\mathrm{Cd}^{2+}$-substituted ${ }^{15} \mathrm{~N}$-labeled $A . v \cdot$ plastocyanin}

$\mathrm{Cd}^{2+}$-substituted ${ }^{15} \mathrm{~N}$-labeled A.v. plastocyanin was prepared as described previously for the copper-containing protein, ${ }^{16}$ except that $\mathrm{CdSO}_{4}$ was added instead of $\mathrm{CuSO}_{4}$. The $\mathrm{Cd}^{2+}$ substituted protein was quantified using the $278 \mathrm{~nm}$ extinction coefficient of $5000 \mathrm{M}^{-1} \mathrm{~cm}^{-1}$ estimated by comparison of the intensities of the NMR signals of copper and cadmium plastocyanins. Freshly purified protein samples were exchanged into $10 \% \mathrm{D}_{2} \mathrm{O} / 90 \% \mathrm{H}_{2} \mathrm{O}, 100 \mathrm{~mm} \mathrm{NaCl}$ and concentrated by ultrafiltration using a stirred Amicon cell fitted with an YM3 membrane.

\section{NMR samples}

The protein concentration in the NMR samples was between 1.0 and $2.7 \mathrm{mM}$, and the $\mathrm{pH}$ was adjusted to 7.0 (meter reading). The NMR sample of the $\mathrm{Cd}^{2+}$-substituted plastocyanin was sealed under nitrogen. Sodium ascorbate $(0.1 \mathrm{~mm})$ was added to the samples of reduced plastocyanin to keep the samples fully reduced, and the NMR tubes were sealed under nitrogen. For the $100 \%$ oxidized plastocyanin samples, a trace of laccase was added to keep the protein fully oxidized and the NMR tubes were sealed under oxygen. All NMR samples used here are listed in Table S1 in 'Supporting information'.

\section{NMR experiments}

The NMR experiments were carried out at $298 \mathrm{~K}$ and ${ }^{1} \mathrm{H}$ frequencies of 500 and $800 \mathrm{MHz}$ using Varian Unity Inova 500 and 800 spectrometers, the former equipped with a cold probe. In all experiments, the ${ }^{1} \mathrm{H}$ carrier was placed on the HDO residual resonance. An overview of the NMR experiments used here along with some of the specific parameters used in the experiments are listed in Table S2 in 'Supporting information'. 
The paramagnetically broadened signals of the protons close to the $\mathrm{Cu}^{2+}$ ion were monitored using a combination of the WEFT ${ }^{13}$ and the TOCSY pulse sequences. As shown previously, ${ }^{17}$ fast repetition of the WEFT pulse sequence, $d_{1}-180^{\circ}-\Delta-90^{\circ}-t_{\mathrm{a}}$, (super-WEFT) allows the suppression of the slow relaxing signals while monitoring only the fast relaxing signals. Thus, the WEFT-TOCSY sequence selectively detects broad resonances by suppressing the sharp resonances. For the WEFT-TOCSY experiment recorded on the $100 \%$ oxidized protein sample, the following delays were used: $\Delta=130 \mathrm{~ms}$ and $t_{\mathrm{a}}+d_{1}=248 \mathrm{~ms}$. Also, a combination of the WEFT sequence and the ${ }^{1} \mathrm{H}-{ }^{15} \mathrm{~N}$ HSQC sequence was applied here with the delays $\Delta=187 \mathrm{~ms}$ and $t_{\mathrm{a}}+d_{1}=302 \mathrm{~ms}$.

Finally, the signal eliminating relaxation filter (SERF) pulse sequence, ${ }^{18} 90^{\circ}-\Delta_{0}-180^{\circ}-\Delta_{1}-180^{\circ}-\Delta_{2}-180^{\circ}-$ $\tau-90^{\circ}-t_{\mathrm{a}}$, in combination with the conventional TOCSY sequence was used in a few cases. The SERF sequence allows a better suppression of the slowly relaxing signals compared with the WEFT sequence, however, at the expense of the signal/noise ratio because of the longer pulse sequence. The SERF delays were, $\Delta_{0}=90 \mathrm{~ms}, \Delta_{1}=163 \mathrm{~ms}, \Delta_{2}=124 \mathrm{~ms}$, $\tau=45 \mathrm{~ms}, t_{\mathrm{a}}=328 \mathrm{~ms}$.

\section{Structure calculations}

The structure calculations were carried out using a version of the Xplor-NIH program, ${ }^{19,20}$ which includes a module that incorporates pseudocontact shifts as restraints in the structure calculations. ${ }^{21}$ The structure calculations were carried out on an Apple XServe G5 cluster with $342.0 \mathrm{GHz}$ processors.

\section{RESULTS AND DISCUSSION}

\section{Spectral assignments}

The assignment of the ${ }^{1} \mathrm{H},{ }^{13} \mathrm{C}$ and ${ }^{15} \mathrm{~N}$ nuclei of reduced A.v. plastocyanin at $\mathrm{pH} 7.0$ and $298 \mathrm{~K}$ was obtained

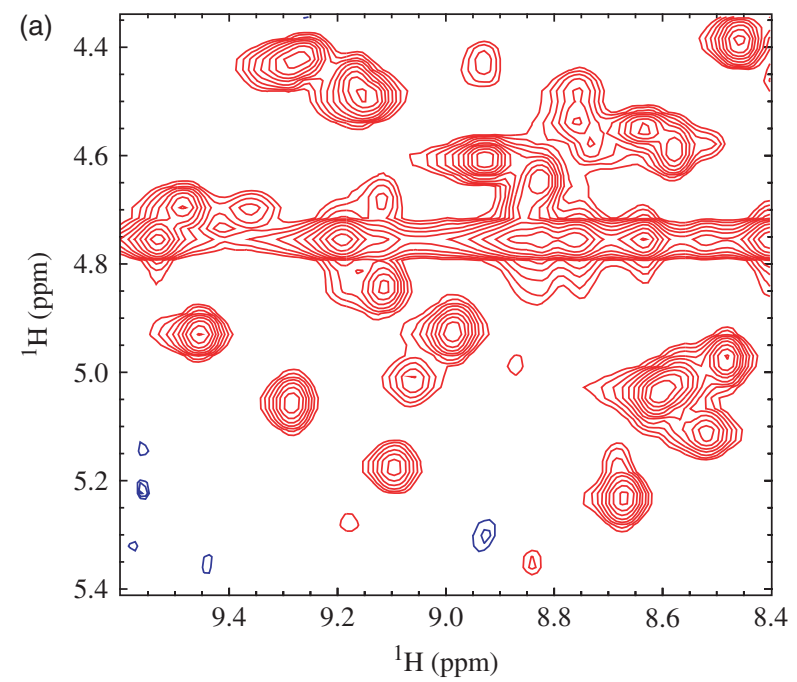

previously. ${ }^{22,23}$ The assignment of the ${ }^{1} \mathrm{H}$ and ${ }^{15} \mathrm{~N}$ nuclei of the $\mathrm{Cd}^{2+}$-substituted plastocyanin under the same conditions was obtained from a ${ }^{1} \mathrm{H}-{ }^{15} \mathrm{~N}$ HSQC spectrum and a conventional TOCSY spectrum, and by comparison with the spectra of the reduced $\mathrm{Cu}^{+}$plastocyanin. The chemical shift values of the ${ }^{1} \mathrm{H}$ and ${ }^{15} \mathrm{~N}$ nuclei in $\mathrm{Cd}^{2+}$-substituted plastocyanin are given in Table S3 in 'Supporting Information'.

The assignment of the signals of the oxidized form of plastocyanin at $\mathrm{pH} 7.0$ and $298 \mathrm{~K}$ was obtained through a comparative analysis of homonuclear and heteronuclear twodimensional NMR spectra. The following two-dimensional spectra of the oxidized form were applied: ${ }^{1} \mathrm{H}-{ }^{15} \mathrm{~N}$ HSQC, ${ }^{1} \mathrm{H}-{ }^{13} \mathrm{C}$ HSQC, TOCSY, WEFT-TOCSY, SERF-TOCSY, and ${ }^{1} \mathrm{H}-{ }^{15} \mathrm{~N}$ WEFT-HSQC. More specifically, the chemical shifts of the amide protons of oxidized plastocyanin were obtained from the ${ }^{1} \mathrm{H}-{ }^{15} \mathrm{~N}$ HSQC spectrum of the protein, while the chemical shifts of the $\alpha$-protons were obtained mainly from the ${ }^{1} \mathrm{H}-{ }^{13} \mathrm{C}$ HSQC spectrum. Additional $\alpha$-proton chemical shifts were obtained from the WEFT-TOCSY and the SERFTOCSY spectra. Figure 1 shows a comparison of the conventional TOCSY spectrum and a WEFT-TOCSY spectrum. As mentioned above, the WEFT sequence effectively suppresses the signals of nuclei with slow longitudinal relaxation rates, while optimizing the spectrum for nuclei with fast longitudinal relaxation rates. Furthermore, in the WEFT-TOCSY spectrum, signals with slow longitudinal relaxation have intensities opposite that of the signals with fast longitudinal relaxation. Consequently, the WEFT-TOCSY experiment makes it possible to identify broad signals of nuclei spatially close to the paramagnetic center and, thereby, to determine pseudocontact shifts in metalloproteins with a relatively long electron relaxation time and relatively small pseudocontact shifts due to a small anisotropy of the $g$ tensor. This is illustrated in Fig. 1, which clearly shows that the WEFT-TOCSY experiment allows the observation of additional paramagnetically broadened signals, such as the signals of N33, H61, and Y88, which are not observed in the

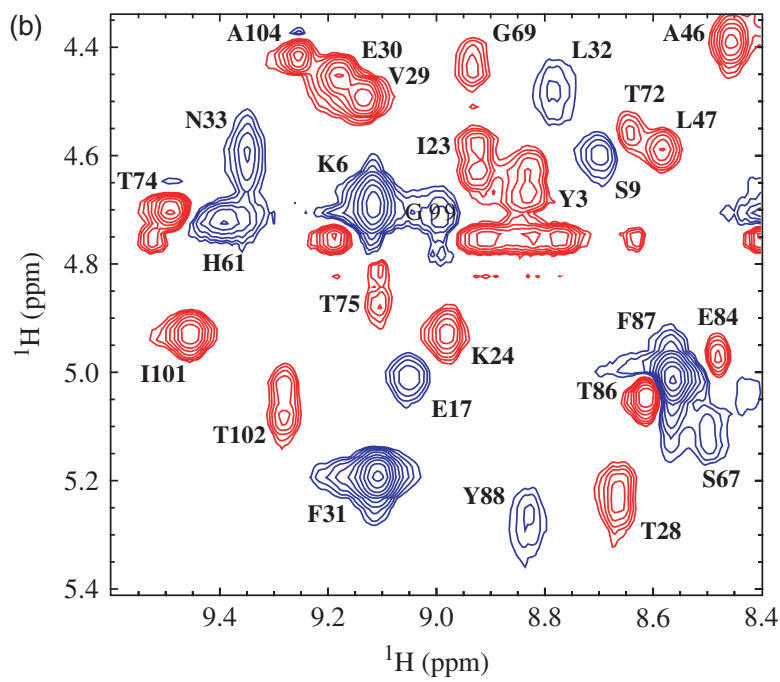

Figure 1. (a) Excerpt of the TOCSY spectrum of a $1.9 \mathrm{~mm}$ sample of $100 \%$ oxidized A.v. plastocyanin at $\mathrm{pH} 7.0$ and $100 \mathrm{~mm} \mathrm{NaCl}$. The excerpt shows the cross peaks between the amide protons and the $\alpha$-protons. (b) Same region of the WEFT-TOCSY spectrum of a $2.7 \mathrm{~mm}$ sample of $100 \%$ oxidized A.v. plastocyanin at pH 7.0 and $100 \mathrm{~mm} \mathrm{NaCl}$. Red contours indicate positive intensity and blue contours indicate negative intensity. 
conventional TOCSY experiment. In particular, suppression of the water signal with the WEFT-TOCSY sequence allows the observation of the H61 signal. The chemical shift values of the ${ }^{1} \mathrm{H}$ and ${ }^{15} \mathrm{~N}$ nuclei in the oxidized A.v. plastocyanin are given in Table S3 in 'Supporting information'.

\section{Chemical shift contributions from intermolecular interactions}

Previously, short-lived transient protein-protein intermolecular interactions were detected in A.v. plastocyanin. ${ }^{24}$ These interactions were revealed by a dependence of the paramagnetic relaxation enhancements of some of the ${ }^{1} \mathrm{H}$ nuclei on the protein concentration. The effect of this intermolecular interaction on the observed pseudocontact shift was investigated here by studying the concentration dependence of the chemical shifts of the amide protons of the oxidized form of plastocyanin. It was found that the chemical shifts of the amide protons remain unchanged as a function of the protein concentration in the range from 0.8 to $2.7 \mathrm{~mm}$ (data not shown). Therefore, the measured pseudocontact shifts arise solely from the dipolar interactions with the unpaired electron of the copper ion within the same protein molecule, while contributions from intermolecular interactions can be neglected.

\section{Choosing a diamagnetic reference}

According to Eqn (1), the pseudocontact shift is obtained as the difference between the chemical shift of the nucleus in the paramagnetic protein and in an appropriate diamagnetic reference, preferably a diamagnetic metal derivative of the same metalloprotein with a close structural resemblance to the paramagnetic protein. Furthermore, the diamagnetic metal ion should have the same charge as the paramagnetic ion in order to avoid chemical shift contributions from a difference in the electric fields in the paramagnetic and the diamagnetic form. In the case of A.v. plastocyanin, the most readily accessible diamagnetic reference is the $\mathrm{Cu}^{+}$protein. Previously, it was shown that $\mathrm{Cu}^{+}$and $\mathrm{Cu}^{2+}$ plastocyanin have nearly identical structures with only minor differences in the first coordination sphere ${ }^{25-27}$ However, because of the different charges of the copper ion in the two forms, also the $\mathrm{Cd}^{2+}$-substituted protein, i.e. $\mathrm{Cd}^{2+}$ A.v. plastocyanin, was used here as a diamagnetic reference. It has been found that the overall structures of $\mathrm{Cd}^{2+}$-substituted blue copper proteins, ${ }^{28,29}$ and other blue copper proteins in which the copper ion is substituted by a different metal ion, e.g. the $\mathrm{Hg}^{2+}$ ion, ${ }^{30}$ are similar to the structures of the coppercontaining proteins.

The effect of the metal ion charge on the chemical shifts is illustrated in Fig. 2, which shows the difference in chemical shifts of the amide protons in $\mathrm{Cu}^{+}$plastocyanin and the $\mathrm{Cd}^{2+}$-substituted protein. As shown in the figure, the change in the electric field caused by the different charges of the metal ions highly influences the chemical shift values. In particular, the amide protons located in the four loop regions surrounding the metal site undergo significant changes in chemical shift, as was also observed previously in the case of pea plastocyanin. ${ }^{31}$ These changes clearly demonstrate the importance of choosing a proper diamagnetic reference.

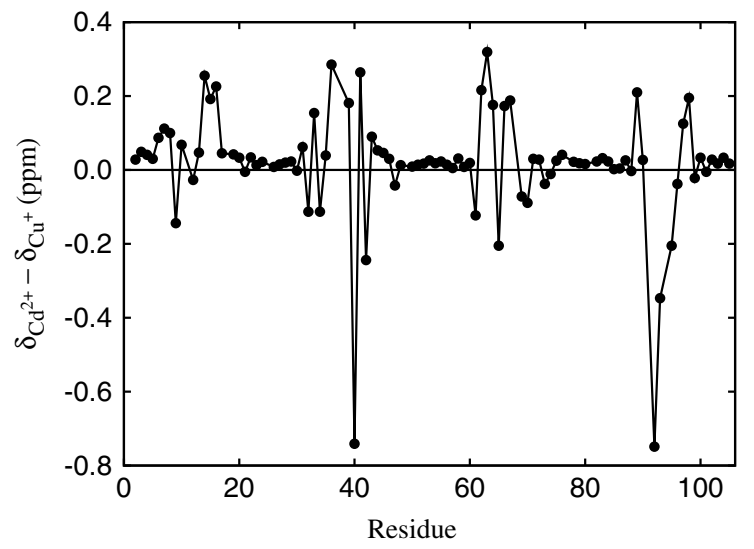

Figure 2. The difference between the chemical shifts of the amide protons in the $\mathrm{Cd}^{2+}$-substituted A.v. plastocyanin and $\mathrm{Cu}^{+}$A.v. plastocyanin as function of the residue number.

\section{Experimental ${ }^{1} \mathrm{H}$ and ${ }^{15} \mathrm{~N}$ pseudocontact shifts}

Two sets of pseudocontact shifts were obtained using either $\mathrm{Cu}^{+}$plastocyanin or $\mathrm{Cd}^{2+}$-substituted plastocyanin as the diamagnetic reference. The pseudocontact shifts were obtained for the amide protons, the $\alpha$-protons and the backbone ${ }^{15} \mathrm{~N}$ nuclei in the protein. A total of $150{ }^{1} \mathrm{H}$ and 79 ${ }^{15} \mathrm{~N}$ pseudocontact shifts were measured.

According to Eqn (2), the pseudocontact shift is independent of the specific type of nucleus and depends only on the position of the nucleus relative to the metal ion. Thus, for an amide group in the protein, similar pseudocontact shifts are expected for the amide proton and the amide ${ }^{15} \mathrm{~N}$ nucleus. However, as pointed out previously, ${ }^{32-34}$ the chemical shifts of the ${ }^{15} \mathrm{~N}$ nuclei are considerably more sensitive than the ${ }^{1} \mathrm{H}$ nuclei to small variations in the backbone torsion angles, the hydrogen bonding and the electric field. Therefore, small local differences in these parameters between the diamagnetic and the paramagnetic form of the protein can compromise the measurement of the pseudocontact shifts of the ${ }^{15} \mathrm{~N}$ nuclei. Erroneous ${ }^{15} \mathrm{~N}$ pseudocontact shifts can be identified by comparing the observed pseudocontact shifts of the amide protons and the backbone ${ }^{15} \mathrm{~N}$ nuclei. Thus, a significant difference ( $>0.2 \mathrm{ppm})$ in the pseudocontact shifts of the two types of nuclei was observed previously in cytochromes and iron-sulfur proteins. ${ }^{35-41}$ As shown in Fig. 3, similar differences are observed for A.v. plastocyanin, independent of whether the $\mathrm{Cu}^{+}$form or the $\mathrm{Cd}^{2+}$-substituted form of the protein is used as the diamagnetic reference. Therefore, the ${ }^{15} \mathrm{~N}$ pseudocontact shifts were not used in the structure refinement of A.v. plastocyanin.

\section{Agreement of the observed ${ }^{1} \mathrm{H}$ pseudocontact shifts with the protein structure}

Initially, the agreement of the observed ${ }^{1} \mathrm{H}$ pseudocontact shifts with the solution structure of A.v. plastocyanin ${ }^{22}$ was investigated. Thus, using the structural coordinates of the protein (PDB: $1 \mathrm{NIN})^{42}$ and the observed ${ }^{1} \mathrm{H}$ pseudocontact shifts, the orientation (the $\alpha, \beta$ and $\gamma$ Euler angles) and the components $\left(g_{\text {ax }}\right.$ and $g_{\text {eq }}$ ) of the anisotropic $g$-tensor were estimated by a least-squares fit of Eqn (2) to the data. Subsequently, the observed pseudocontact shifts were 

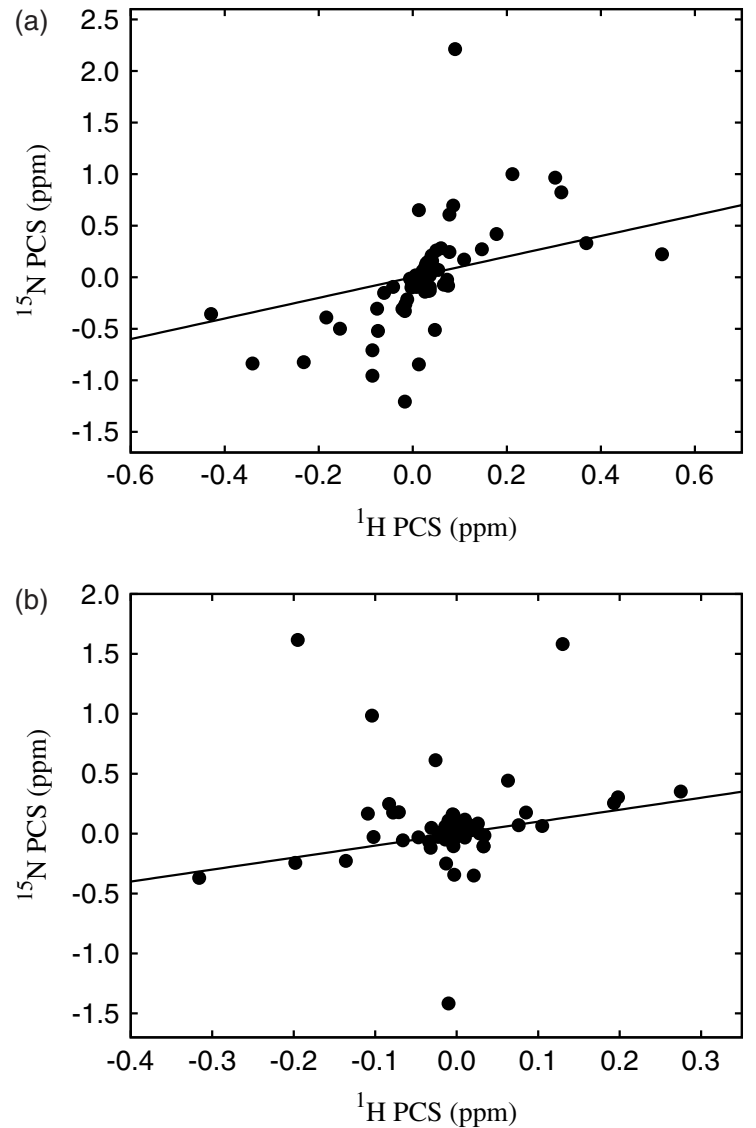

Figure 3. Comparison of the experimental pseudocontact shifts (PCS) of the amide protons and amide ${ }^{15} \mathrm{~N}$ nuclei in $\mathrm{Cu}^{2+}$ A.v. plastocyanin obtained using $\mathrm{Cu}^{+}$plastocyanin (a) and $\mathrm{Cd}^{2+}$-substituted plastocyanin (b) as the diamagnetic reference.

compared with the corresponding shifts calculated from the $g$-tensor parameters obtained in the least-squares fit and the solution structure, ${ }^{22}$ as shown in Fig. 4 . The leastsquares fit was carried out using the pseudocontact shifts obtained with both $\mathrm{Cu}^{+}$plastocyanin (a) and the $\mathrm{Cd}^{2+}$ substituted plastocyanin (b) as the diamagnetic reference. The anisotropic $g$-tensor components obtained in the two cases were, $g_{\text {ax }}=0.752 \pm 0.053, g_{\text {eq }}=-0.255 \pm 0.069\left(\mathrm{Cu}^{+}\right)$ and $g_{\mathrm{ax}}=0.535 \pm 0.029, g_{\mathrm{eq}}=-0.100 \pm 0.036\left(\mathrm{Cd}^{2+}\right)$. Furthermore, in both cases $g_{z z}$ is oriented about $10^{\circ}$ away from the $\mathrm{Cu}-\mathrm{S}$ (Met97) bond. These values should be compared with the principal $g$ values obtained previously for spinach plastocyanin using EPR, ${ }^{43}$ i.e. $g_{x x}=2.042, g_{y y}=2.059$, and $g_{z z}=2.226$, and a tilt angle of $g_{z z}$ relative to the $\mathrm{Cu}-\mathrm{S}$ (Met97) bond of about $5^{\circ}$. According to Eqns (3) and (4), the three $g$ values correspond to the $g$-tensor components, $g_{\text {ax }}=0.750$ and $g_{\text {eq }}=-0.070$.

Figure 4 shows that the agreement between the observed and the calculated pseudocontact shifts is only modest, although the agreement appears slightly better when the $\mathrm{Cd}^{2+}$-substituted plastocyanin is used as the diamagnetic reference. This apparent disagreement between the two sets of pseudocontact shifts could be due to a relatively low precision of the solution structure of the protein caused by an insufficient number of NOE restraints. This
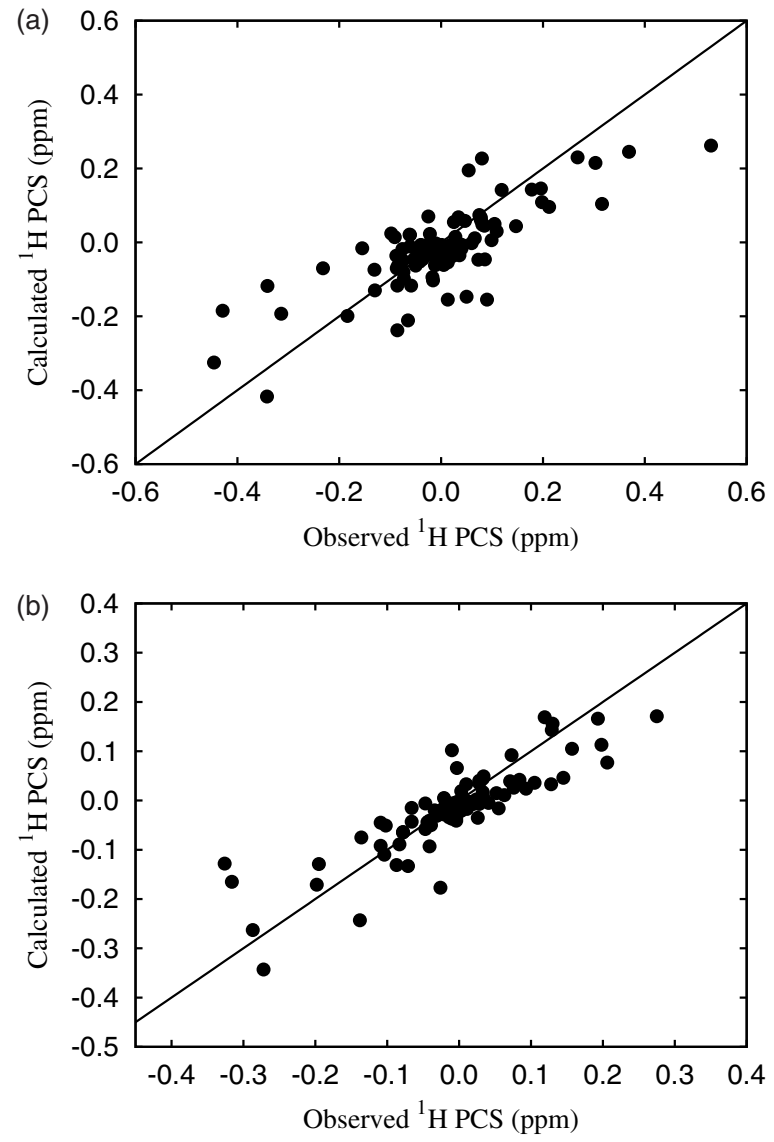

Figure 4. Comparison of the observed and the calculated ${ }^{1} \mathrm{H}$ pseudocontact shifts based on the solution structure of $A . v$. plastocyanin. ${ }^{22}$ The calculated pseudocontact shifts were obtained from the solution structure and the $g$-tensor parameters, derived as described in the text using Eqn (2), the solution structure, and the experimental pseudocontact shifts. In (a), the $\mathrm{Cu}^{+}$plastocyanin was used as the diamagnetic reference. In (b), the $\mathrm{Cd}^{2+}$-substituted plastocyanin was used as the diamagnetic reference.

is further supported by a comparison with the crystal structure of Phormidium laminosum (P.l.) plastocyanin ${ }^{44}$ (PDB: $\left.1 \mathrm{BAW}^{42}\right)$, a plastocyanin that is, a close homologue to A.v. plastocyanin ( $66 \%$ sequence identity). The crystal structure of P.l. plastocyanin at $2.7 \AA$ resolution was solved by molecular replacement using the unpublished crystal structure of A.v. plastocyanin at $1.7 \AA$ resolution as a search model. ${ }^{44}$ Figure 5 shows a superposition of the backbones (residues 1-105) of the crystal structure of P.l. plastocyanin (red trace) and the solution structure of $A$. $v$. plastocyanin (green trace). The RMSD between the backbones of the two structures is $1.62 \AA$. Figure 6 shows a comparison of the observed and calculated pseudocontact shifts based on the crystal structure of P.l. plastocyanin. Again, a leastsquares fit was carried out using both $\mathrm{Cu}^{+}$plastocyanin (a) and $\mathrm{Cd}^{2+}$-substituted plastocyanin (b) as the diamagnetic reference. The obtained $g$-tensor parameters in the two cases were, $g_{\mathrm{ax}}=1.037 \pm 0.069, g_{\text {eq }}=-0.238 \pm 0.077\left(\mathrm{Cu}^{+}\right)$and $g_{\mathrm{ax}}=0.841 \pm 0.032, g_{\text {eq }}=-0.048 \pm 0.035\left(\mathrm{Cd}^{2+}\right)$. In both cases $g_{z z}$ is oriented about $9^{\circ}$ away from the $\mathrm{Cu}-\mathrm{S}($ Met97) bond. Interestingly, a good agreement between the observed 


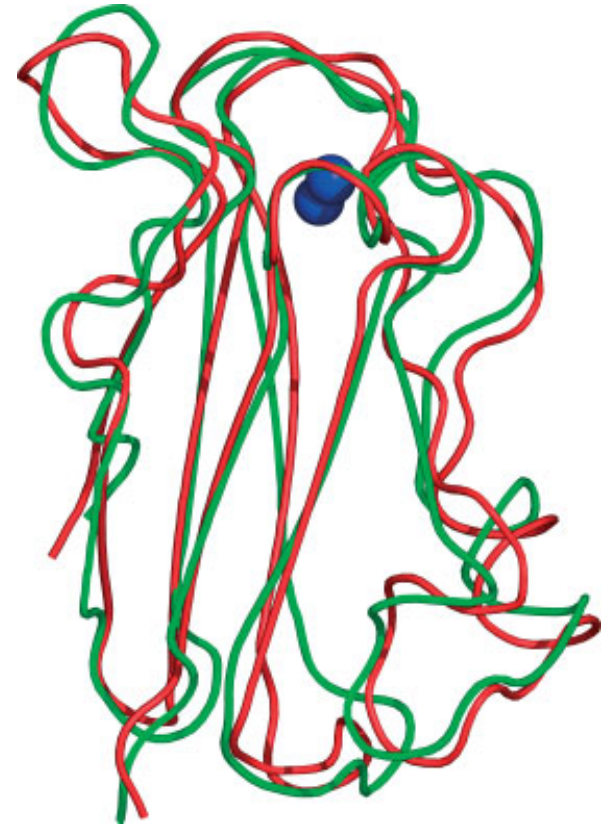

Figure 5. Superposition of the crystal structure of $P . I$. plastocyanin ${ }^{44}$ (red trace) and the solution structure of $A$. $v$. plastocyanin $^{22}$ (green trace). The blue spheres indicate the copper ions. The figure was prepared with PyMOL. ${ }^{45}$.

and calculated pseudocontact shifts is now obtained when the $\mathrm{Cd}^{2+}$-substituted protein is used as a diamagnetic reference. Also, the size and the orientation of the $g$-tensor obtained in the least-squares fit are in good agreement with the experimental values obtained previously for plastocyanin from spinach ${ }^{43}$ (see above). In contrast, a rather poor agreement is seen when the $\mathrm{Cu}^{+}$protein is used as the diamagnetic reference. These observations clearly indicate that the $\mathrm{Cd}^{2+}$-substituted plastocyanin is a more appropriate diamagnetic reference than $\mathrm{Cu}^{+}$plastocyanin. As mentioned above, this can be explained by the identical charges of the diamagnetic $\mathrm{Cd}^{2+}$ ion and the paramagnetic $\mathrm{Cu}^{2+}$ ion. At the same time, the agreement of the pseudocontact shifts from A.v. plastocyanin with the structure of P.l. plastocyanin support the above suggestion that the solution structure of A.v. plastocyanin ${ }^{22}$ is less precise, and that a more precise solution structure can be obtained by including the measured pseudocontact shifts as restraints in the structure refinement. As indicated by the similarity of the solution structure of A.v. plastocyanin and the crystal structure of P.l. plastocyanin (Fig. 5), only minor changes of the solution structure will lead to a better agreement between the observed and calculated pseudocontact shifts. Thus, the long-range pseudocontact shifts may be sensitive to minor inaccuracies in the protein structure that are not easily revealed by the conventional NOE restraints.

\section{Structure refinement}

The refinement of the solution structure of A.v. plastocyanin was carried out using the structure calculation program Xplor-NIH. ${ }^{19,20}$ The ${ }^{1} \mathrm{H}$ pseudocontact shifts obtained using the $\mathrm{Cd}^{2+}$-substituted protein as the diamagnetic reference were included as restraints in the structure refinement by
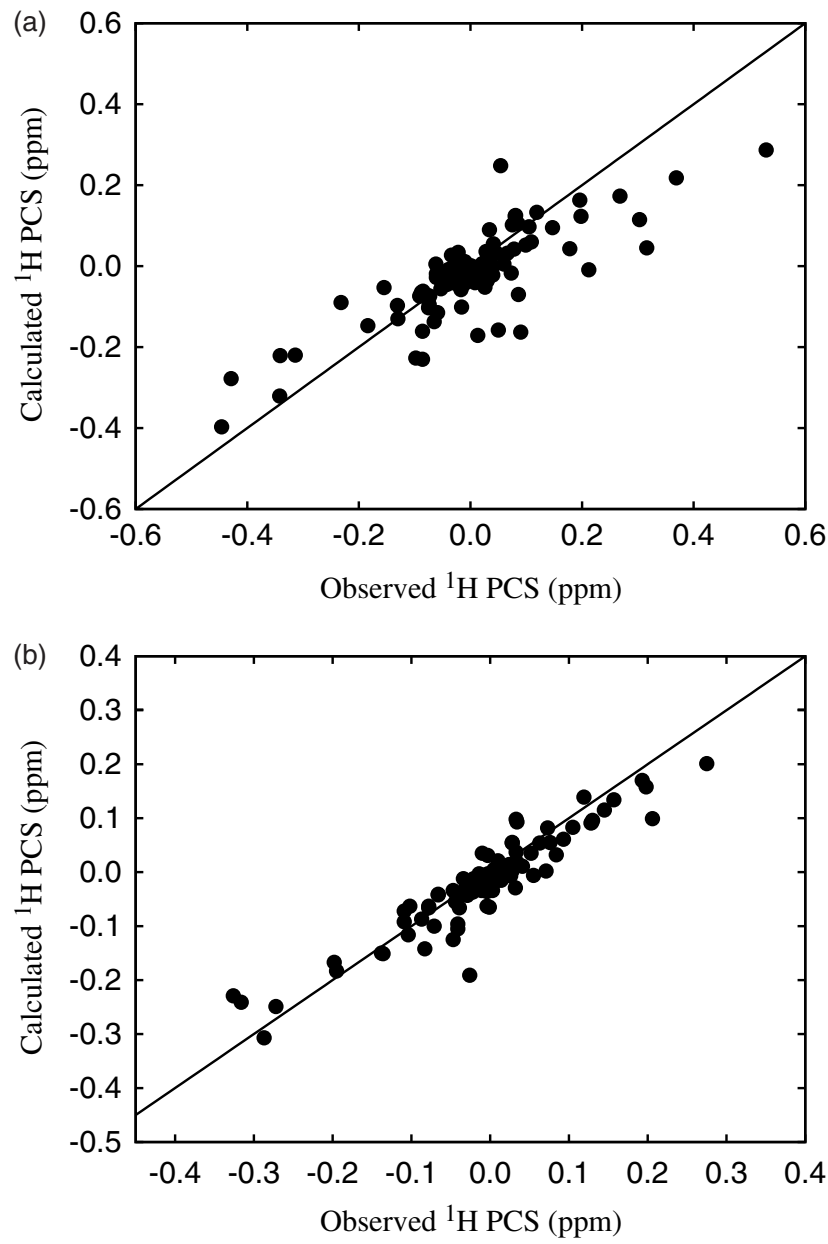

Figure 6. Comparison of the observed and the calculated ${ }^{1} \mathrm{H}$ pseudocontact shifts based on the crystal structure of P.I. plastocyanin. ${ }^{44}$ The calculated pseudocontact shifts were obtained from the crystal structure of P.I. plastocyanin, and the $g$-tensor parameters, derived as described in the text using Eqn (2), the crystal structure and the experimental pseudocontact shifts. In (a), the $\mathrm{Cu}^{+}$plastocyanin was used as the diamagnetic reference. In (b), the $\mathrm{Cd}^{2+}$-substituted plastocyanin was used as the diamagnetic reference.

applying the module PARArestraints available for XplorNIH. ${ }^{21}$ The module takes into account the pseudocontact shifts through the energy penalty function ${ }^{21}$

$$
E=k_{\text {force }} \sum_{i}\left[\max \left(\left|\mathrm{PCS}_{i, \text { obs }}-\mathrm{PCS}_{i, \text { calc }}\right|-\text { tol }_{i}, 0\right)\right]^{2}
$$

Here, $k_{\text {force }}$ is the force constant, PCS $_{i, \text { obs }}$ are the observed pseudocontact shifts, $\mathrm{PCS}_{i, \text { calc }}$ are the calculated pseudocontact shifts based on the protein structure and the $g$-tensor, and tol $_{i}$ is the uncertainty of the observed pseudocontact shifts.

Essentially, the structure refinement was carried out as described in the example input file saCONV.inp. ${ }^{21}$ Thus, the set of NOEs and dihedral angles ${ }^{22}$ were included in the refinement of the solution structure of A.v. plastocyanin obtained previously. ${ }^{22}$ A total of 50 structures were calculated using the experimental pseudocontact shifts, the initial values of the $g$-tensor parameters, $g_{\mathrm{ax}}=1.72$ and $g_{\text {eq }}=-1.15$, and a force constant of $100 \mathrm{kcal} \mathrm{mol}^{-1} \mathrm{ppm}^{-2}$. Subsequently, 
the $g$-tensor parameters were calculated from the $20 \%$ lowest energy subset of the 50 structures. These parameters were used in the next iteration where again 50 structures were calculated. A total of ten iterations of the $g$-tensor parameters were performed to ensure a complete convergence.

Figure 7 shows the iterative convergence of the $g$-tensor parameters obtained from a fit of the tensor parameters to the total data set of pseudocontact shifts. The final set of values were $g_{\mathrm{ax}}=0.689$ and $g_{\mathrm{eq}}=-0.149$. It is seen that the value of $g_{\text {ax }}$ obtained here is in good agreement with the value obtained previously by EPR, ${ }^{43}$ while the value of $g_{\text {eq }}$ is slightly larger (numerically) than was determined by EPR. In the lowest energy structure, $g_{z z}$ is oriented $6^{\circ}$ away from the $\mathrm{Cu}-\mathrm{S}(\mathrm{Met} 97)$ bond. Figure 8 shows a comparison of the observed and calculated pseudocontact shifts obtained in the structure refinement. As it appears from the figure, the agreement between the two sets of pseudocontact shifts is now good.

Figure 9 shows 'sausage' diagrams (ten structures with lowest total energy) of the structure obtained from the NOE restraints alone (a) and the refined structure where also the ${ }^{1} \mathrm{H}$ pseudocontact shifts were included in the structure determination (b). The backbone RMSD between the ten structures is $0.86 \AA$ before the refinement and $0.75 \AA$ after the refinement, indicating an improvement in the precision of the solution structure when the pseudocontact shifts are included as restraints. In particular, the pseudocontact shifts result in a better determination of the structure in loop regions close to the metal site where only few NOE restraints are available. The PROCHECK ${ }^{46}$ Ramachandran analysis was carried out using the ten structures with lowest energy. The results before refinement were $53.5 \%$ (most favored regions), 43.0\% (additional allowed regions), $3.5 \%$ (generously allowed regions), and $0.0 \%$ (disallowed regions). After refinement with pseudocontact shifts, the results were $55.8 \%$ (most favored regions), $40.7 \%$ (additional allowed regions), 3.5\% (generously allowed regions), and $0.0 \%$ (disallowed regions).

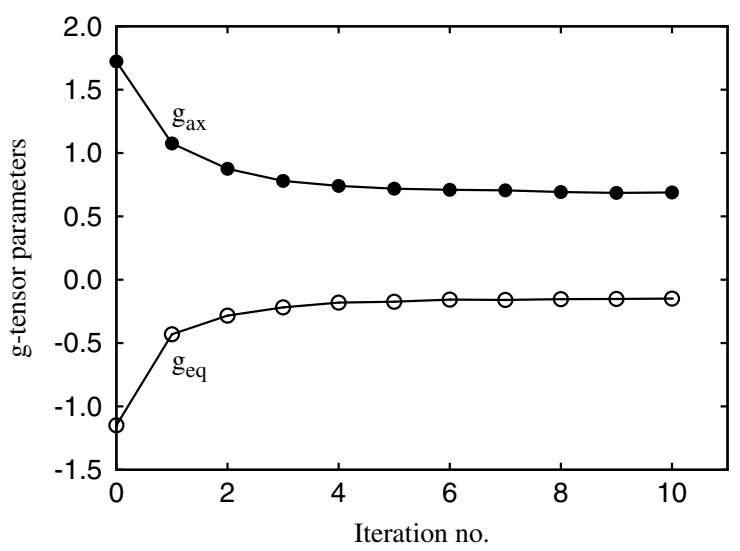

Figure 7. The iterative convergence of the anisotropic $g$-tensor components, $g_{\mathrm{ax}}$ and $g_{\mathrm{eq}}$, obtained using the module PARArestraints for Xplor-NIH. ${ }^{21}$ The initial values of the tensor parameters were $g_{\mathrm{ax}}=1.72$ and $g_{\mathrm{eq}}=-1.15$, while the force constant used for the pseudocontact shift restraints was $100 \mathrm{kcal} \mathrm{mol}^{-1} \mathrm{ppm}^{-2}$.

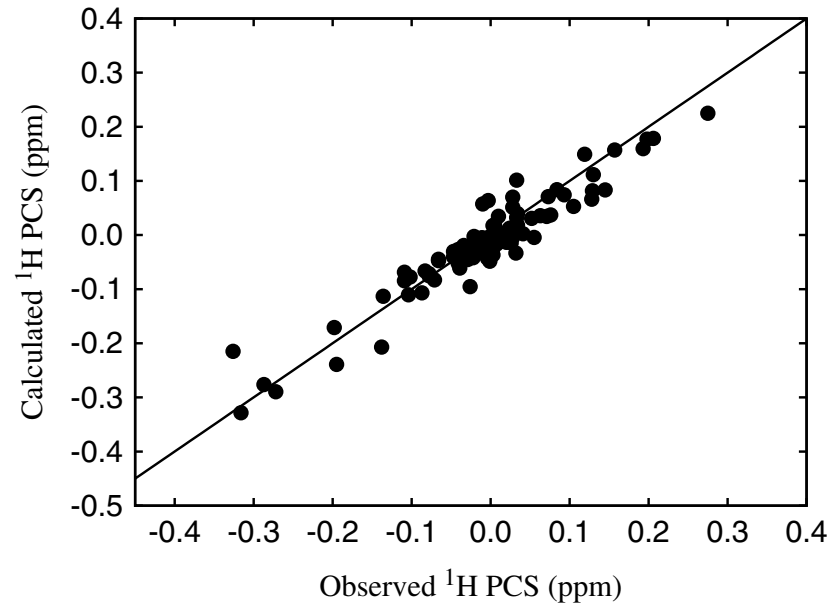

Figure 8. Comparison of the observed and calculated ${ }^{1} \mathrm{H}$ pseudocontact shifts of the solution structure with the lowest energy obtained in the structure refinement. The calculated pseudocontact shifts were obtained by the module PARArestraints for Xplor-NIH. ${ }^{21}$
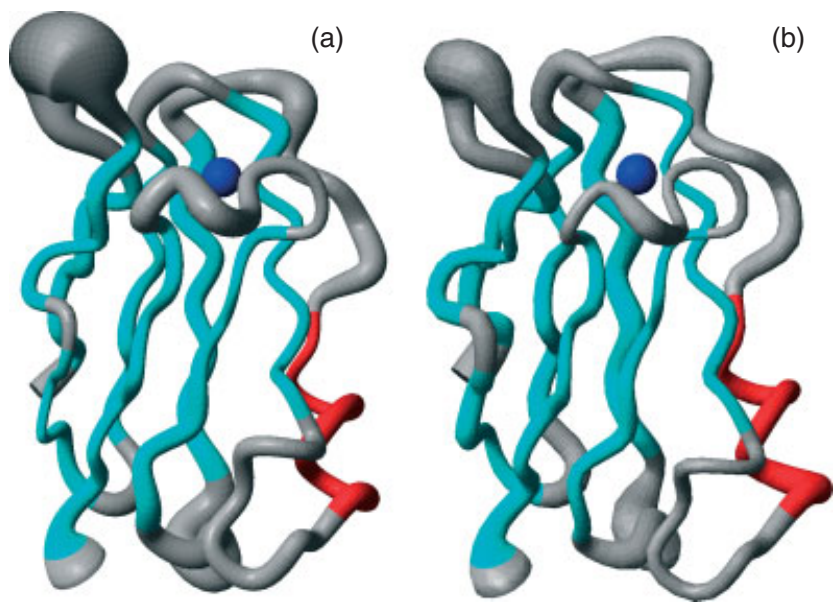

Figure 9. (a) A 'sausage' diagram of the ten structures with lowest total energy of the solution structure of A.v. plastocyanin obtained using conventional NOEs and dihedral angle restraints. ${ }^{22}$ (b) A 'sausage' diagram of the ten structures with lowest total energy obtained in the structure refinement of the solution structure of A.v. plastocyanin where also the ${ }^{1} \mathrm{H}$ pseudocontact shifts were included as restraints. In both figures, the blue sphere indicates the copper atom. The figure was prepared with MOLMOL. ${ }^{47}$

\section{CONCLUSION}

In conclusion, it has been shown that pseudocontact shifts of ${ }^{1} \mathrm{H}$ nuclei relatively close to the paramagnetic metal ion can be determined accurately, using the WEFT pulse sequence in combination with the TOCSY or the ${ }^{1} \mathrm{H}-{ }^{15} \mathrm{~N}$ HSQC sequences. As demonstrated here, this holds even for a copper protein, where the signals are broad because of a relatively long electron relaxation time, and the pseudocontact shifts are small because of a relatively small anisotropy of the $g$-tensor. Furthermore, the importance of choosing an appropriate diamagnetic reference has been investigated, and the influence on the chemical shifts 
of the electrical charge of the metal ion is emphasized. Specifically, it is found that $\mathrm{Cd}^{2+}$-substituted plastocyanin is a more suitable diamagnetic reference as compared with the diamagnetic $\mathrm{Cu}^{+}$-plastocyanin, due to an electrical charge similar to that of the copper ion in the paramagnetic $\mathrm{Cu}^{2+}$ plastocyanin. A structure refinement of plastocyanin that includes ${ }^{1} \mathrm{H}$ pseudocontact shifts as restraints, in addition to the conventional NOEs and dihedral angle restraints, improves the precision of the protein solution structure, in particular, in loop regions close to the metal site where the number of conventional NOE restraints is limited. In general, the study indicates that the long-range pseudocontact shifts are sensitive to minor inaccuracies in protein structures that are not easily revealed by the conventional NOE restraints.

\section{Acknowledgements}

We thank Gabriele Cavallaro, Charles Schwieters, and Søren M. Kristensen for helpful discussions, and Else Philipp, Jens Duus, and Bent $\mathrm{O}$. Petersen for technical assistance. The $800 \mathrm{MHz}$ spectra were acquired at the Danish Instrument Center for NMR Spectroscopy of Biological Macromolecules.

\section{REFERENCES}

1. Gryk MR, Finucane MD, Zheng Z, Jardetzky O. J. Mol. Biol. 1995; 246: 618.

2. Zheng Z, Gryk MR, Finucane MD, Jardetzky O. J. Magn. Reson., Ser. B 1995; 108: 220 .

3. Jensen MR, Petersen G, Lauritzen C, Pedersen J, Led JJ. Biochemistry 2005; 44: 11014.

4. Wüthrich K. NMR of Proteins and Nucleic Acids. John Wiley \& Sons: New York, 1986.

5. Gochin M J. Biomol. NMR 1998; 12: 243.

6. Tu K, Gochin M J. Am. Chem. Soc. 1999; 121: 9276.

7. Allegrozzi M, Bertini I, Janik MBL, Lee YM, Liu G, Luchinat C. J. Am. Chem. Soc. 2000; 122: 4154.

8. Gochin M. Structure 2000; 8: 441.

9. Bertini I, Janik MBL, Lee YM, Luchinat C, Rosato A. J. Am. Chem. Soc. 2001; 123: 4181.

10. Bertini I, Donaire A, Jiménez B, Luchinat C, Parigi G, Piccioli M, Poggi L. J. Biomol. NMR 2001; 21: 85.

11. Gaponenko V, Sarma SP, Altieri AS, Horita DA, Li J, Byrd RA. J. Biomol. NMR 2004; 28: 205.

12. Ubbink M, Ejdebäck M, Karlsson BG, Bendall DS. Structure 1998; 6: 323.

13. Patt SL, Sykes BD. J. Chem. Phys. 1972; 56: 3182.

14. Bertini I, Luchinat C. Coord. Chem. Rev. 1996; 150: 131.

15. Kurland RJ, McGarvey BR. J. Magn. Reson. 1970; 2: 286.

16. Hass MAS, Thuesen MH, Christensen HEM, Led JJ. J. Am. Chem. Soc. 2004; 126: 753

17. Inubushi T, Becker ED. J. Magn. Reson. 1983; 51: 128.
18. Hansen DF, Led JJ. J. Magn. Reson. 2001; 151: 339.

19. Brünger AT. XPLOR - A System for X-Ray Crystallography and NMR, Version 3.1. Yale University Press: New Haven, 1992.

20. Schwieters CD, Kuszewski JJ, Tjandra N, Clore GM. J. Magn. Reson. 2003; 160: 65.

21. Banci L, Bertini I, Cavallaro G, Giachetti A, Luchinat C, Parigi G. J. Biomol. NMR 2004; 28: 249.

22. Badsberg U, Jørgensen AMM, Gesmar H, Led JJ, Hammerstad JM, Jespersen LL, Ulstrup J. Biochemistry 1996; 35: 7021.

23. Ma L, Hass MAS, Vierick N, Kristensen SM, Ulstrup J, Led JJ. Biochemistry 2003; 42: 320.

24. Hansen DF, Hass MAS, Christensen HM, Ulstrup J, Led JJ. J. Am. Chem. Soc. 2003; 125: 6858.

25. Guss JM, Harrowell PR, Murata M, Norris VA, Freeman HC. J. Mol. Biol. 1986; 192: 361.

26. Bertini I, Bryant DA, Ciurli S, Dikiy A, Fernández CO, Luchinat C, Safarov N, Vila AJ, Zhao J. J. Biol. Chem. 2001; 276: 47217.

27. Bertini I, Ciurli S, Dikiy A, Fernández CO, Luchinat $\mathrm{C}$, Safarov N, Shumilin S, Vila AJ. J. Am. Chem. Soc. 2001; 123: 2405.

28. Engeseth HR, McMillin DR, Otvos JD. J. Biol. Chem. 1984; 259: 4822.

29. Blackwell KA, Anderson BF, Baker EN. Acta Crystallogr. 1994; D50: 263.

30. Church WB, Guss JM, Potter JJ, Freeman HC. J. Biol. Chem. 1986; 261: 234.

31. Ubbink M, Lian LY, ModiS, Evans PA, Bendall DS. Eur. J. Biochem. 1996; 242: 132.

32. de Dios AC, Pearson JG, Oldfield E. Science 1993; 260: 1491.

33. Le H, Oldfield E. J. Phys. Chem. 1996; 100: 16423.

34. Neal S, Nip AM, Zhang H, Wishart DS. J. Biomol. NMR 2003; 26: 215.

35. Guiles RD, Basus VJ, Sarma S, Malpure S, Fox KM, Kuntz ID, Waskell L. Biochemistry 1993; 32: 8329.

36. Bertini I, Dikiy A, Kastrau DHW, Luchinat C, Sompornpisut P. Biochemistry 1995; 34: 9851.

37. Boyd J, Dobson CM, Morar AS, Williams RJP, Pielak GJ. J. Am. Chem. Soc. 1999; 121: 9247.

38. Morelli X, Dolla A, Toci R, Guerlesquin F. Eur. J. Biochem. 1999; 261: 398.

39. Tsan P, Caffrey M, Daku ML, Cusanovich M, Marion D, Gans P. J. Am. Chem. Soc. 1999; 121: 1795.

40. Bertini I, Luchinat C, Turano P. J. Biol. Inorg. Chem. 2000; 5: 761.

41. Lehmann T, Luchinat C, Piccioli M. Inorg. Chem. 2002; 41: 1679.

42. Berman HM, Westbrook J, Feng Z, Gilliland G, Bhat TN, Weissig H, Shindyalov IN, Bourne PE. Nucleic Acids Res. 2000; 28: 235.

43. Penfield KW, Gewirth AA, Solomon EI. J. Am. Chem. Soc. 1985; 107: 4519 .

44. Bond CS, Bendall DS, Freeman HC, Guss JM, Howe CJ, Wagner MJ, Wilce MCJ. Acta Cryst. 1999; D55: 414.

45. DeLano WL. The PyMOL Molecular Graphics System. DeLano Scientific: San Carlos, CA, 2002.

46. Laskowski RA, MacArthur MW, Moss DS, Thornton JM. J. Appl. Crystallogr. 1993; 26: 283.

47. Koradi R, Billeter M, Wüthrich K. J. Mol. Graphics 1996; 14: 51.

\section{SUPPORTING INFORMATION}

Tables listing the NMR samples used and the NMR experiments performed are available. Also, a table containing the chemical shift values of the ${ }^{1} \mathrm{H}$ and ${ }^{15} \mathrm{~N}$ nuclei of $\mathrm{Cd}^{2+}$-substituted A.v. plastocyanin and the paramagnetic $\mathrm{Cu}^{2+} A . v$. plastocyanin is available at http:/ / www.spectroscopyNOW.com/nmr/supplementary 